\title{
Tobacco smoking in persons with schizophrenia followed up at a teaching hospital in Sri Lanka
}

\author{
N Edrisinghe, CA Wijesinghe, SS Williams, KALA Kuruppuarachchi
}

\section{Background}

Studies in Western populations indicate that the prevalence of smoking in patients with schizophrenia ranges from $70-90 \%$. Data from low and middle income countries is sparse.

Aims

To determine the prevalence of smoking in patients with schizophrenia, and to identify probable associations.

\section{Methodology}

The study was conducted at the psychiatry outpatient clinics of Colombo North Teaching Hospital, Ragama. Consecutive outpatients who met ICD-10 diagnostic criteria for schizophrenia were invited to answer an interviewer administered, pre-tested questionnaire on socio-demographic status, smoking habits, disease and treatment related characteristics. The Fagerstrom's Test for Nicotine Dependence was used to grade the level of nicotine addiction.

\section{Results}

Of the 400 patients with schizophrenia, 171 (42.8\%) were smokers. Of them 150 (87.7\%) were dependent on nicotine. In terms of Fagerstrom's Test, high depen- dence was seen in $3.3 \%(n=5)$ while $39.2 \%(n=59)$ and $57.3 \%(n=86)$ reported moderate and low dependence respectively. All the smokers were male. For a majority of the patients the age of initiation of smoking was between 16-25 years, correlating with the onset of schizophrenia. Interestingly, most of the smokers (63.2\%) were keen to quit smoking. Participants who smoked were significantly more likely to misuse other substances $(p=0.001)$ and to suffer from physical illnesses ( $p=0.0001)$, compared to non-smokers. Smokers were also significantly more likely to be on intramuscular depot antipsychotics $(p=0.032)$, whereas non-smokers in contrast, were more likely to be on atypical antipsychotics ( $p=0.0001)$.

\section{Conclusion}

A dual diagnosis of schizophrenia and nicotine dependence could be made in nearly half of our study population with schizophrenia. Although these rates are lower than in western populations, it is an issue that needs to be addressed in the treatment.

Key words: smoking, schizophrenia, nicotine dependence, substance misuse

SL J Psychiatry 2014; 5(2) 19-23

\section{Introduction}

According to the World Health Organisation (WHO), there are more than one billion smokers worldwide. It is estimated that tobacco use will cause one billion deaths worldwide during the 21st century, with three quarters of these deaths occurring in low-income countries (1). A study in the UK showed that smoking is the single most common cause of death and that half of all smokers will die prematurely because of their addiction (2). The overall life expectancy of a smoker is on average 8 years less than that of a non-smoker (2). Smoking is a major risk factor for at least 20 diseases including coronary and peripheral vascular disease, chronic bronchitis and at least $80 \%$ of lung cancers (2).

A majority of those with major mental illnesses are also known to smoke. Studies show that up to $70 \%$ of individuals with schizophrenia smoke cigarettes (3). It is highlighted that a majority of those with schizophrenia who smoke, started smoking before the onset of the illness. Patients who smoked were younger males and they required more frequent hospitalisations and greater contact with psychiatric services compared to nonsmokers. In addition, many were receiving intramuscular antipsychotic medication (4). Some studies have postulated that one of the major reasons for excess natural mortality among patients with schizophrenia is cigarette smoking (5). A comprehensive meta-analysis highlighted that smoking can be regarded as a plausible explanation for the raised mortality from respiratory disease in this population (6). The question has been raised whether the widespread smoking behaviour seen in this population is in fact a manifestation of an underlying psychological need, that drives these patients to smoke in an attempt to self medicate (7). Evidence suggests that smoking may bring about a reduction in psychiatric symptoms and antipsychotic induced side effects, as well as an enhancement of attention, working memory and other cognitive functions (7). Smoking is further 
known to improve processing of auditory stimuli (sensory gating) in patients with schizophrenia and to lessen negative symptoms by increasing dopamine in the nucleus accumbens and the prefrontal and frontal cortex (8).

Some western studies show that use of traditional antipsychotics may result in patients smoking more, whereas patients taking atypical antipsychotics may smoke less. Further, patients who smoke metabolize antipsychotics faster than non-smokers (8). Smoking is also considered a risk factor for the development of metabolic syndrome in patients who are prescribed antipsychotics (8).

Most studies suggest that almost two-thirds of patients with schizophrenia are smokers; nicotine dependence appears to be by far the most important dual diagnosis in this patient population (9). Other than the associated excess morbidity and mortality, the financial cost associated with smoking in patients with schizophrenia has already been identified. Most of these people are also unemployed due to their illness and this double blow of smoking and unemployment hits the patients very hard. (10). It is also known that smokers are more likely than non-smokers to use or abuse other psychoactive substances. Further, people with schizophrenia and substance misuse have poor symptomatic and functional outcome than those with schizophrenia alone.

Therefore, it is clear that smoking affects all aspects of life in a person with schizophrenia. Despite the high prevalence of smoking in patients with schizophrenia, it is often under-diagnosed and inadequately addressed. Generally, service systems managing schizophrenia and nicotine dependence tend to be separated, with poor intercommunication (11). Effective management of these disorders, however, requires an integrated approach because of the close interrelationship between these disorders.

Most data on this topic is derived from research conducted in western countries, except for a few studies from countries such as India (12). To the best of our knowledge, there is no research data on the prevalence of nicotine dependency in patients with schizophrenia in Sri Lanka.

Considering all these issues, the objectives of this study was to estimate the prevalence of smoking and it's associations among patients with schizophrenia who were followed up at a teaching hospital in Sri Lanka.

\section{Methods}

This was a cross sectional descriptive study, carried out at the psychiatry clinics of the Professorial Psychiatry Unit, Colombo North Teaching Hospital, Ragama, Sri Lanka. The eligible study population comprised all persons over 16 years of age who were diagnosed to have schizophrenia, who attended the clinics from 1st September 2009 to 28th February 2010. Those who had difficulty in understanding the questions due to severe cognitive deficits or florid psychotic symptoms were excluded. All eligible individuals were invited to participate. Recruitment process was continued until the pre-planned number of participants was reached.

Ethical clearance was obtained from the ethics review committee of the Faculty of Medicine, University of Kelaniya, Sri Lanka.

Data was collected from each participant via an interviewer-administered questionnaire. Outcomes of interest included socio-demographic details, details of smoking, co-morbid psychoactive substance misuse and nature of the schizophrenic illness and treatment.

The Fragerstrom's Questionnaire (FNTD) was used to assess the degree of nicotine dependence. The interview was conducted using the language which was most convenient to the patient, by multilingual research assistants. Statistical analysis was carried out using SPSS version 15 for Windows and Chi-Square test was administered for categorical data.

\section{Results}

\section{Demographic details}

Our study sample consisted of 400 participants of whom $79.2 \%(n=317)$ were males. The mean age of the participants was $39.9(\mathrm{SD}=11.1$ ) years. The educational background of the participants included $42.8 \%(n=171)$ with formal education, of which $35.8 \%(n=143)$ had studied up to grade $5-10$, and $15.5 \%$ ( $n=86)$ had studied up to the GCE A/L or University. There were no professionals among the participants. Just above half the sample (52.2\%) were unmarried. Only a small number (5.3\%) was divorced, widowed or separated. The majority of participants (53.5\%) were unemployed while about (18.5\%) were engaged in skilled work. The monthly income ranged from less than 5000 Rupees (57.2\%) to more than 25,000 Rupees (2.5\%).

\section{Smoking prevalence and level of nicotine dependence}

In this study sample $42.8 \%(n=171)$ of persons with schizophrenia were current smokers. As per the Fagerstrom's Test for Nicotine Dependence, of the 171 smokers $87.7 \%(n=150)$ were dependent on nicotine. There was high dependence identified in 3.3\% $(n=5)$, moderate dependence in $39.2 \%(n=59)$ and low dependence in $57.3 \%(n=86)$. Half $(50 \%, n=86)$ of the smokers reported smoking in order to reduce boredom and $19.9 \%,(n=34)$ said they smoked to improve their mood, while $16.4 \%$ ( $n=28 / 171)$ reported no specific reason. Interestingly a majority $(63.2 \%, n=108)$ of smokers said they wanted to quit the habit.

\section{Co-morbid psychoactive substance misuse}

Of all the participants, $41 \%(n=163)$ were misusing other substances. Among them 65.7\% ( $\mathrm{n}=107)$ misused alcohol, 
25.1\% ( $n=41)$ used cannabis, and 9.2\% $(n=15)$ took two or more substances. The smokers $(81.8 \%, n=140 / 171)$ were significantly more likely to misuse other substances than non- smokers $(10.4 \%, n=23)(p<0.01)$.

\section{Smoking and its association with illness}

Most of the sample (49.2\%) had experienced the first episode of schizophrenia between 16-25 years of age; and a majority $79.5 \%(n=136)$ initiated smoking at the same age. With regards to other illness - 29.4\% $(n=116)$ of participants were found to have comorbid physical illness such as hypertension (24\%), diabetes mellitus (18\%), ischaemic heart disease $(11 \%)$ or chronic obstructive airway disease (21\%). Patients with schizophrenia who smoked (45.6\%, $n=78$ ) were significantly more likely to have physical illness than the non-smoking patients $(16.5 \%, n=38)(p<0.01)$.

\section{Effect of smoking on treatment}

Atypical antipsychotics were prescribed for $44.3 \%$ ( $n=177)$ of participants, while 37\% $(n=148)$ were on typical antipsychotics only. A combination of typical and atypical were being used by $18.7 \%(n=75)$. Table 1 illustrates association between smoking and medication use.

\section{Discussion}

\section{Prevalence of smoking in schizophrenia}

It has been reported that the prevalence of smoking is modestly higher in urban populations $(29.9 \%)$ than in rural areas (24.4\%) of Sri Lanka (13). Considering that our study population is from Ragama, a semi-urban setting, a similar prevalence could be expected. But the findings of this study show a much higher proportion of smokers $42.8 \%$ ( $n=171)$ among patients with schizophrenia than in the general population of Sri Lanka.
However the prevalence of smoking in this study sample was much lower than reported rates for smoking in patients with schizophrenia in most western countries. The prevalence of smoking in patients with schizophrenia in south-west Scotland has been reported to be 58\% (4), while in the United States of Americas a prevalence of $88 \%$ was identified $(4,14)$. Interestingly the prevalence of smoking seen in this study was broadly similar to that reported in an Indian study (15). Therefore the socioeconomic setting of the population may be an equally important determinant when considering the high prevalence of smoking in patients with schizophrenia.

\section{Level of nicotine dependence}

In a similar study conducted in India it was shown that, $63 \%$ of males, and $80 \%$ of females had moderate to severe nicotine dependence (12). The severity of nicotine dependence was lower among our participants with only 3.5\% being highly dependent while $39.2 \%$ and $57.3 \%$ had moderate and low levels of dependency respectively. In addition all of them were males. This may have been influenced by financial constraints and the negative attitude among families towards smoking in Sri Lanka.

\section{The role of gender and smoking in schizophrenia}

The gender rates with regards to smoking in schizophrenia differ markedly in studies conducted worldwide. Some studies report that only a mild increase in smoking is seen in male patients with schizophrenia compared to female patients (9). Other studies have concluded that there was no gender difference in the patterns of nicotine use among patients with psychiatric illnesses (16). On the contrary, a study from India has noted a marked gender difference in tobacco use among patients with psychiatric illnesses (17). In our sample all patients with schizophrenia who smoked were males. None of the female participants had smoked. The gender difference

\begin{tabular}{|c|c|c|c|c|c|c|c|}
\hline \multirow[t]{2}{*}{$\begin{array}{l}\text { Type of antipsychotic } \\
\text { Medication }\end{array}$} & \multicolumn{2}{|c|}{$\begin{array}{l}\text { Smokers } \\
(n=171)\end{array}$} & \multicolumn{2}{|c|}{$\begin{array}{l}\text { Non-smokers } \\
(n=229)\end{array}$} & \multirow[t]{2}{*}{$d f$} & \multirow[t]{2}{*}{$x^{2}$} & \multirow[t]{2}{*}{$P$ value } \\
\hline & $\mathrm{N}$ & $\%$ & $\mathrm{~N}$ & $\%$ & & & \\
\hline Typical oral only & 45 & 26.3 & 38 & 16.5 & 1 & 5.627 & 0.017 \\
\hline Typical depot only & 6 & 3.5 & 2 & 0.8 & 1 & 3.469 & 0.062 \\
\hline Typical oral + typical depot & 35 & 20.4 & 22 & 9.6 & 1 & 9.45 & 0.002 \\
\hline Atypical oral only & 54 & 31.5 & 123 & 53.7 & 1 & 19.44 & 0.00001 \\
\hline Typical oral + atypical oral & 11 & 6.4 & 13 & 5.6 & 1 & 0.099 & 0.753 \\
\hline Typical depot + atypical oral & 16 & 9.3 & 28 & 12.2 & 1 & 1.072 & 0.30 \\
\hline $\begin{array}{l}\text { Typical oral + typical depot }+ \\
\text { Atypical oral }\end{array}$ & 3 & 1.7 & 3 & 1.3 & 1 & 0.131 & 0.717 \\
\hline Any depot antipsychotics & 61 & 35.7 & 59 & 25.7 & 1 & 4.577 & 0.032 \\
\hline
\end{tabular}


observed in our study was more marked than both Western and Indian studies. This may reflect the social and cultural norms in Sri Lanka.

\section{Smoking and its psychological effect}

Some studies have reported that smoking in schizophrenia was strongly associated with subjective effects of cheerfulness, agility, alertness, concentration and calmness, with the latter being the most important reason quoted for its usage when compared to the normative population (18). In contrast, the majority of patients in this study reported that they smoked in order to reduce boredom.

\section{Smoking and other substance misuse}

Co-morbid psychiatric and substance use disorders present significant challenges and have a negative impact on treatment adherence, treatment response, course and outcome of both conditions and lead to increased service utilisation, morbidity and mortality. Anumber of epidemiological surveys over the past two decades have consistently reported that individuals with psychiatric disorders have higher rates of substance use disorders, and those who have substance use disorders have higher rates of psychiatric disorders $(18,19)$. Alcohol, cannabis, cocaine and nicotine are the primary substances of abuse in schizophrenia $(18,19)$. It is also postulated that smokers are more likely to misuse other psychoactive substances than non-smokers. The findings in our study sample resemble those observed in western studies; other substance misuse including alcohol and cannabis was significantly more common among smokers than non-smokers, highlighting the substantial burden on service providers and families.

\section{Smoking and physical comorbidity}

Although only $29.4 \%$ of our participants had other comorbid illness, there was a significant association between physical comorbidities in patients with schizophrenia who smoked, compared to non-smokers. This is supported by previous similar evidence. It has been reported that people with schizophrenia have higher rates of cardiovascular disease, including myocardial infarction, than the general population (20). Patients with schizophrenia are more likely than the general population to have lifestyle risk factors for cardiovascular disease including smoking $(6,10,21,22)$. Costs associated with tobacco use may also increase economic hardships and thus further negatively impact physical health (10).

\section{Smoking and antipsychotic medication}

It has been reported that smokers were more likely to be receiving intramuscular typical antipsychotic medication and less likely to be receiving oral and atypical antipsychotics $(23,24)$. This corresponded to findings in our study as well. However it is not clear whether atypical antipsychotic use is effective in reducing smoking rates in schizophrenia, as studies in western populations where patients are more likely to be on atypical antipsychotics show higher rates of smoking than of our study population. The fact that smokers in our study were more likely to be on depot antipsychotics than non-smokers hints at a possible association between smoking and drug compliance.

\section{Limitations}

We investigated a limited geographical area in the Western province, which may not be representative of Sri Lanka in all aspects. The exclusion of patients with cognitive impairment or florid psychotic symptoms may have lowered the prevalence rates of smoking as such patients are more likely to smoke. Data related to smoking habits was based only on self-reporting in the absence of facilities to measure blood or urine nicotine levels, which may have impacted on the findings. Absence of a control group prevents the direct comparison with the general population.

\section{Conclusions}

A dual diagnosis of schizophrenia and nicotine dependence can be made in nearly half of our study population with schizophrenia. Although these rates are lower than in western populations, it is an issue that needs to be addressed in the management of these individuals. In Sri Lanka, this is a problem that appears to effect chiefly male patients, and to be associated with the use of typical antipsychotics and depot medications. Further research is needed to explore effective and culturally suitable strategies to reduce rates of smoking among this patient group.

\section{Declaration of interest \\ None declared}

\section{N Edrisinghe, CA Wijesinghe, SS Williams, KALA Kuruppuarachchi, University Psychiatry Unit, Faculty of Medicine, University of Kelaniya.}

\section{Corresponding author: $\mathrm{N}$ Edrisinghe}

Email: edirisinghe.nayana06@gmail.com

\section{References}

1. World Health Organization (WHO). Prevention of mental disorders: effective interventions and policy options. Geneva: WHO; 2004.

2. Callum C. The UK Smoking Epidemic; Deaths in 1995. London: Health Education Authority; 1998.

3. Hughes JR, Hatssukami DK, Mitchel JE, Dahlgren LA. Prevalence of smoking among psychiatric outpatients. Am J psychiatry 1986;143: 993-7. 
4. Ciara K, McCreadie RG. Smoking Habits, Current Symptoms, and Premorbid Characteristics of Schizophrenic Patients in Nithsdale, Scotland. Am J psychiatry 1999; 156(11): 1751-7.

5. Brown S, Inskip H, Barraclough B. Causes of the excess mortality of schizophrenia. Br J Psychiatry 2000; 177: 212-7.

6. Brown S. Excess mortality of schizophrenia - A meta analysis. Br J Psychiatry 1997; 171: 502-8.

7. Kumari V, Postma P. Nicotine use in Schizophrenia - The self medication hypothesis. Neurosci Behav Rev 2005; 29(6): 1021-4.

8. Edward RL. A Review of the effects of the nicotine on schizophrenia and antipsychotic medications. Psychiatr Serv 1999; 50: 1346-50.

9. McCreadie RG. Use of drugs, alcohol and tobacco by people with schizophrenia - case control study. Br J Psychiatry 2002; 81: 321-5.

10. Robin G, McCreadie RG, Ciara K. Patients with schizophrenia who smoke - Private disaster, public resource. Br J Psychiatry 2000; 176: 109.

11. Kavanagh DJ, John S, John B, et al. Substance Misuse in patients with Schizophrenia. Epidemiol Manag 2002; 62(5): 743-55.

12. Chandra PS, Carey MP, Carey KB, Jairam KR, Rudresh HP. Prevalence and Correlates of Tobacco Use and Nicotine Dependence among Psychiatric Patients in India. Addict Behav 2005; 30(7): 1290-99.

13. Silva VD, Samarasinghe D, Gunawardane N. Alcohol and Tobacco Use among males in two districts in Sri Lanka. Ceylon Med J 2009; 54(4):119-24.

14. Hughes JR, Hatsukami DK, Mitchell JE, Dahlgren LA. Prevalence of smoking among psychiatric outpatients. Am J Psychiatry 1986; 143: 993-7.
15. Srinivasan TN, Parthasarathy K. Smoking Pattern in Patients with Mental Disorders - Observation in A Developing Country. India: Schizophrenia Research Foundation; 1998.

16. Farrell M, Howes S, Bebbington $P$, et al. Nicotine, alcohol and drug dependence and psychiatric comorbidity. $\mathrm{Br} \mathrm{J}$ Psychiatry 2001; 179: 432-7.

17. Gurpegui M, Martinez - Ortega JM, Jurado D, et al. Subjective effects and the main reason for smoking in outpatients with schizophrenia - case control study. Compr Psychiatry 2007; 48: 86-91.

18. Regier DA, Farmer MC, Rae DS, et al. Co morbidity of Mental Disorders with Alcohol and other drug abuse Results from Epidemiologic Catchment Area (ECA) Study. JAMA 1990; 264: 2511-8.

19. Kessler RC, Crum RM, Warner LA, Nelson CB. Lifetime co-occurance of DSM lll-R alcohol abuse and dependence with other Psychiatric Disorders in National Community Survey. Arch of Gen Psychiatry 1997; 313-21.

20. Tsung MT, Perkins K, Simpson JC. Physical Disorders in schizophrenia and affective disorders. J Clin Psychiatr 1983; 44: 42-6.

21. Jeste DV, Gladsjo JA, Lindamer LA, Lacro JP. Medical comorbidity in schizophrenia. Schizophr Bull 1996; 22: 413-36.

22. Kendrick T. Cardiovascular and respiratory risk factors and symptoms among general practice patients with longterm Mental Illness. Br J Psychiatry 1996; 169: 733-9.

23. Kelly C, McCreadie RG, McEwan T, et al. Nithsdale schizophrenia surveys 17 - Fifteen year review. Br J Psychiatry 1998; 172: 513-7.

24. McEvoy JP, Freudenreich O, Levin ED, Rose JE. Haloperidol increases smoking in patients with schizophrenia. Psychopharmacology 1995; 119: 124-6. 